\title{
Thy1 (+) and (-) lung fibrosis subpopulations in LEW and F344 rats
}

\author{
J.C. Mclntosh*, J.S. Hagood**, T.L. Richardson**, J.W. Simecka ${ }^{+}$
}

Thyl (+) and (-) lung fibrosis subpopulations in LEW and F344 rats. J.C. McIntosh, J.S. Hagood, T.L. Richardson, J.W. Simecka. CERS Journals Ltd 1994.

ABSTRACT: Appreciation of the potential of fibroblasts as effector cells in inflammation has led to the recognition of fibroblast subpopulations, the most stable of which are the Thy1 (+) and Thy1 (-) subpopulations in mouse lung fibroblasts. We investigated the presence of Thy $1(+)$ and (-) fibroblasts in rats, comparing the percentage in primary cultures from rats with different susceptibility to fibrosis, and whether the characteristics were similar in mice and rats, and between normal and fibrotic rats lungs.

Using primary cultures of rat fibroblasts obtained both from normal and fibrotic lungs, we analysed the percentage of Thy1 (+) and (-) fibroblasts by fluorescenceactivated cell sorter (FACS) analysis. We sorted the fibroblasts to evaluate immune region associated antigen (Ia) expression, which tends to be raised in tissues involved in inflammation, and other characteristics.

We found that Thy1 (+) and (-) fibroblasts: 1) are distinct subpopulations in rat lungs; 2) are found in different proportions in rat strains with different propensity towards lung fibrosis; and 3) have similar but not identical characteristics in mice and rats. We also found that bleomycin-induced fibrosis increases the percentage of Ia expression in Thy1 (-), but not Thy1 (+) fibroblasts.

The presence of these stable fibroblast subpopulations in multiple species, and the fact that these fibroblasts differ in their response to a fibrosing agent, suggests the importance of considering fibroblast subpopulations in development and disease.

Eur Respir J., 1994, 7, 2131-2138.
*Dept of Pediatric Pulmonary Disease, Duke University Medical Center, Durham, NC, USA. Depts of **Pediatric Pulmonary Disease and +Microbiology, University of Alabama at Birmingham, Alabama, USA.

Correspondence: J.C. McIntosh

Dept of Pediatrics

Division of Pediatric Pulmonary Disease Box \# 2994, Rm 302 Bell Bldg

Duke University Medical Center Durham, NC 27710

USA

Keywords: Fibroblasts fibroblast subpopulations pulmonary fibrosis

Received: November 251993 Accepted after revision August 251994
There is a clear, but complex inter-relationship between fibroblasts, inflammation, and pulmonary fibrosis. Current theories implicate inflammation as the driving force that leads to fibrosis [1]. An insult initiates the inflammatory cascade which is essential to the appropriate immune response for host defence. Usually, the response then abates and normality is restored. However, if inflammation continues in an uncontrolled manner, it can lead to recruitment, proliferation, and stimulation of many types of cells, including fibroblasts, resulting in damage and scarring of the lungs. As responder cells, fibroblasts are the key producers of matrix materials, such as collagen and fibronectin. Fibroblasts are also capable of acting as effector cells, actively influencing the inflammatory cascade as well as responding to it.

Fibroblasts are key cells in determining whether the outcome of the host response to an insult is a return to normality or progression to fibrosis. Inflammation is an essential component, but even in diseases marked by intense inflammation, such as adult respiratory distress syndrome (ARDS), fibrosis is not inevitable [2]. The factors determining tissue remodelling following inflammation, either into repair or fibrosis, are, for the most part, unknown. Other cells, such as the alveolar macrophage, type II pneumocytes and inflammatory cells are clearly also of importance, but fibroblasts are unique in their ability to act as both responder and effector cells. Lung fibroblasts produce matrix components (collagen and fibronectin) and cytokines that promote inflammation (interleukins-1, -6 and -8 (IL-1, IL-6, and IL-8)) [3, 4], and they can promote repair by their interactions with type II pneumocytes [5, 6]. Fibroblasts can also prolong the survival of inflammatory cells in vitro $[7,8]$. Thus, fibroblasts play a multifaceted role in inflammation and repair.

To add to the complexity of these interactions, there is clear evidence that fibroblasts are not a homogenous population. Functional differences, such as differing response to various modifiers of growth and collagen production, are seen in fibroblasts derived from different tissues [9], fibroblasts from the same organ cultured from healthy and diseased individuals, and in different clones originating from the same diseased tissue [10, 11]. Phenotypic differences have also been described. Subpopulations of rat lung fibroblasts differ in the predominant expression of collagen types (I and III) [12], and 
foetal rat lung fibroblasts differ in their predominant form of glycosaminoglycan production [13], and intracellular lipid content [14]. Human lung fibroblasts exist in high and low C1q binding subpopulations [15]. The most stable phenotype difference so far described in lung fibroblasts is the expression of Thy 1 on fibroblast subpopulations, as described in mouse lungs by PHIPPs et al. [16]. These fibroblast subpopulations are quite stable. There is no cross-over in over 30 passages in either species, whereas the other means of distinguishing subpopulations tend to be lost over time, either because of maturational effects lipid containing interstitial cell (LIC fibroblasts) [14] or "reversion to the mean" in culture.

In vitro, Thy1 (+) and (-) fibroblasts have divergent capacities to influence the direction of inflammation. Thy 1 is a member of the immunoglobulin superfamily, which, in general, are antigens involved in intracellular interactions [17]. By this fact alone, it seems likely that fibroblasts that express Thy1 (i.e. Thy1 (+) fibroblasts) would possess more potential for paracrine interactions than those which do not (i.e. Thy1 (-) fibroblasts). There is, at present, no proof to this assertion, but there is the observation that Thy1 (+) fibroblasts appear to have more cell-to-cell contacts in vitro than do Thy1 (-) fibroblasts [18]. If that contact is with epithelial cells, Thy1 (+) fibroblasts could promote repair by enhancing type II pneumocyte hyperplasia, a role in repair that fibroblasts clearly play [5]. On the otherhand, Thy1 (-) fibroblasts were shown by PHIPPs et al. [16] to be capable of expressing factors that would be considered proinflammatory. Thy1 (-) fibroblasts can produce interleukin (IL)-1 $\alpha$, whereas Thy1 (+) fibroblasts cannot [19]. IL1 , both $\alpha$ and $\beta$, are cytokines thought to be important in initiating the inflammatory cascade. Only Thy 1 (-) fibroblasts were shown to be able to express immune region associated antigen (Ia), the rodent major histocompatibility complex (MHC) II antigen [16]. Ia expression is necessary for antigen presentation, and tends to be elevated in tissues involved in inflammation. Thus, Ia expression and IL- $1 \alpha$ production suggest a role for Thy1 (-) fibroblasts in promoting or perpetuating inflammation.

We have worked with Lewis (LEW/NCr (LEW)) and Fisher (F344/NCr (F344)) rats in evaluating lung fibrosis. We have characterized a model of airway fibrosis following infection with Mycoplasma pulmonis, and have shown that LEW rats develop more severe airway fibrosis than do F344 rats [20]. We have also shown that LEW rats develop more severe interstitial fibrosis following instillation of bleomycin sulphate [21]. This relationship of one strain having more severe pulmonary fibrosis following two unrelated insults is unique. We hypothesized that this difference in fibrotic potential may, in part, be explained by differences in the stromal composition. Based on the observations by PHIPPS et al. [16] that Thy 1 $(+)$ and (-) fibroblasts differentially influence inflammation, we sought to determine if there were Thy1 (+) and (-) lung fibroblast subpopulations in naive animals from both strains. We examined whether BLM-induced fibrosis would change the characteristics of those fibroblasts.
We report here our findings that both of these rat strains do have lung fibroblast subpopulations differentiated by Thy 1 expression, that LEW rats (the "fibrosis prone" strain) have a higher percentage of Thy1 (-) fibroblasts than F344 rats, that Thy1 (+) and (-) fibroblasts in rats and mice have similar characteristics, and that Thy $1(-)$ fibroblasts have a higher percentage of Ia expression after BLM, but Thy1 (+) fibroblasts do not.

\section{Materials and methods}

\section{Animals}

Six week old ( \pm 1 week), pathogen-free LEW and F344 rats, either reared at the University of Alabama at Birmingham (UAB), or obtained from the National Cancer Institute (Frederick, Md, USA) and maintained in Trexlertype plastic film isolators, were used. The pathogen-free status of the animal colonies was monitored [17]. $\mathrm{A} \mathrm{CO}_{2}$ chamber was used to sacrifice the animals. All procedures employed were approved by the UAB at Animal Welfare Assurance committee (assurance number A3255-01), a fully accredited American Association For Accreditation of Laboratory Animal Care (AAALAC) institution.

\section{Bleomycin administration}

To examine "activated" lung fibroblasts (i.e. those derived from diseased lung tissue), bleomycin sulphate (BLM) was instilled intratracheally following anaesthetic by methoxyflurane (Metofane, Pittman-Moore, Mundelein, IL, USA) inhalation. When the animal no longer resisted manipulation, but was maintaining adequate respiration, the trachea was exposed and BLM $\left(10 \mathrm{U} \cdot \mathrm{kg}^{-1}\right)$ was directly instilled. The wound was closed with 4-0 Ethilon and the animal allowed to recover. Five days later, the animals were sacrificed by overexposure in the $\mathrm{CO}_{2}$ chamber and the lungs processed as below. Slightly outdated bleomycin sulphate (Blexane) was kindly provided by D.L. Elliott, Bristol-Meyers Pharmaceuticals (Evansville, Indiana, USA).

\section{Establishing primary cultures of lung fibroblasts}

The chest wall of the rats were opened under sterile conditions, their right ventricles cannulated, and the pulmonary vasculature perfused with phosphate buffered saline (PBS) until clear. The heart and lungs were then removed en bloc, the lung lobes separated, minced to approximately $0.5 \mathrm{~mm}$ pieces, and then suspended in 10 $\mathrm{ml}$ of digestion medium comprised of RPMI 1640 (Gibco, Grand Island, NY, USA) with the following: $0.05 \%$ trypsin (Sigma, St. Louis), $0.005 \%$ deoxyribonuclease (DNase) (Sigma), $25 \mu \mathrm{M}$ hydroxyethylpiperazine ethanesulphonic acid (HEPES) (Gibco), pH 7.4.

After $10 \mathrm{~min}$, the digestion was stopped by the addition of an equal volume of bovine calf serum (BCS), and 
then washed three times with RPMI with 5\% BCS (Whittaker, Walkerville, MD, USA). The tissue was disaggregated by filtration through sterile nylon mesh (150 $\mu \mathrm{M})$, washed by centrifugation in PBS, and suspended in growth medium, consisting of RPMI with the following: $10 \%$ foetal bovine serum (FBS) (Whittaker), $2 \mu \mathrm{M} \mathrm{L}-$ glutamine (Gibco), $25 \mu \mathrm{M}$ HEPES, 1\% antibiotic/antimycotic mixture (Gibco), 0.0025\% gentamicin (Gibco). The resulting cell suspensions were placed into T-25 flasks for $4 \mathrm{~h}$ in a $\mathrm{CO}_{2}$ incubator, and then washed gently with RPMI to remove nonadherent cells. Growth medium was added $(5.5 \mathrm{ml})$ and exchanged every 2-3 days. In general, the initial split was accomplished between 5-9 days after culturing the cells. That split and all subsequent divisions were made 1:3. Once the cells were in log-phase growth, the flasks were split every 3-5 days.

\section{Antibody labelling and fluorescence-activated cell sorter (FACS) analysis}

Confluent fibroblasts were dislodged using gentle trypsinization. (Note: consistent with the data from PHIPPS et al. [16], we have repeatedly shown that both Thy 1 and Ia expression persist after trypsin digestion of cells). Cells, $1 \times 10^{6}$, were washed three times in PBS with $2 \%$ FBS and $0.1 \% \mathrm{NaN}_{3}$ (Sigma), and then centrifuged at $4^{\circ} \mathrm{C}$ for $10 \mathrm{~min}$ at $500 \times \mathrm{g}$. The tubes were decanted and blotted after the third wash. They were then incubated with the primary antibody (labelled with marker) in the appropriate concentration and for the established length of time. Control cells were simultaneously incubated with a secondary antibody with the same marker. Cells were then washed three times, blotted, resuspended in $0.5 \mathrm{ml}$ of PBS with $1 \%$ paraformaldehyde, and kept in foil at $4^{\circ} \mathrm{C}$ until analysis. FACS analysis was performed by K. Berry (University of Alabama Flow Cytometry Core Facility, Division of Clinical Immunology and Rheumatology, NIH AR 20614).

We used this procedure for two different experiments. Firstly, to determine the presence of Thy1 (+) and (-) subpopulations and to evaluate whether the percentage of these lung fibroblast subpopulations in primary culture differed between LEW and F344 rat strains. We took six rats from each strain and cultured the lung fibroblasts. We performed FACS analysis on low passage (4th first experiment, and 3rd second experiment) fibroblasts using a mouse anti-rat (MAR) Thy1 antibody (OX7) with a fluorescein isothiocyanate label (FITC), (kindly supplied by J. Eldridge, Dept of Microbiology, UAB), which was added to the cells (1:80 dilution, $\left.20 \mu \mathrm{l} \cdot \mathrm{vial}^{-1}\right)$. Control cells were suspended in rat anti-mouse (RAM) FITC (R \& D Systems, Minneapolis, MN, USA) at the manufacturer's specified dilution (20 $\mu \mathrm{l}$ of 1:50 dilution). All cells were incubated at $4^{\circ} \mathrm{C}$ for $30 \mathrm{~min}$. The cells with FITC staining above controls were considered positive (i.e Thy $1(+)$ ), and the percentage positive cells compared between the stains. This experiment was performed twice.

Secondly, sorted lung fibroblasts were evaluated for the expression of Ia (rodent MHC class II antigen). Four groups of sorted cells (Thy1 (+) and (-) fibroblasts from naive and BLM exposed rats) were seeded into $75 \mathrm{~cm}^{2}$ flasks $\left(7.5 \times 10^{5}\right.$ cells $)$ in standard growth medium alone (one flask of each subpopulation) or with interferon (IFN)$\gamma\left(500 \mathrm{U} \cdot \mathrm{ml}^{-1}\right.$ added daily for 4 days, two flasks of each subpopulation). (IFN- $\gamma$ was a generous gift from Genentech, South San Francisco, USA). The purity of each culture was checked using FACS analysis before utilization in any assay. Only flasks with greater than $90 \%$ purity were used. After $96 \mathrm{~h}$, the cells were split and analysed for Ia expression (OX-3 with phycoerythrin (PE) label, R \& D Systems) or with PE ( $R$ \& D Systems) control antibody, diluted according to manufacturer's specifications. Incubation was for $1 \mathrm{~h}$, and controls for each subpopulation were used to allow for differences in nonspecific binding of PE. Cells with higher than control PE labelling were considered Ia positive. This procedure was performed three times, and the data of the groups were compiled for statistical analysis and graphic presentation.

\section{FACS sorting into Thyl (+) and (-) groups}

The protocol was identical to that for Thy1 staining except that azide was not used and sterility was maintained. Approximately $6 \times 10^{6}$ cells were labelled with OX-7/FITC, and sorted by intensity of FITC labelling. The lowest $25 \%$ of the negative cells and the highest $25 \%$ of the positive cells were sorted for a minimum of $2.5 \times 10^{5}$ cells. The sorted cells were plated in T-25 flasks in growth medium. After three passages, a repeat sorting was performed, using the same parameters. A third sorting was needed for the cells derived from BLM recipient animals.

\section{Immunohistochemical staining for collagen}

Sorted fibroblasts were seeded sparsely $\left(20,000\right.$ cells.well-1 $\left.{ }^{-1}\right)$ in standard growth medium into four 8-chamber slides (Lab-Tek, Naperville, IL, USA) in a fashion that would allow randomization. After seeding, the cells were incubated in growth medium containing $0.05 \%$ ascorbic acid and $0.05 \% \beta$-aminopropionitrile for $48 \mathrm{~h}$. The following solutions were used in the procedure: PBSTween (100 $\mathrm{\mu l}$ Tween 20 in $200 \mathrm{ml}$ PBS), Tris buffer $\left(0.145 \mathrm{M} \mathrm{NaCl}, 0.025 \mathrm{M}\right.$ Tris- $\mathrm{HCl}, 0.02 \% \mathrm{NaN}_{3}$ at a $\mathrm{pH}$ of 7.6) and activating solution (10 mg of 3,3'-diaminobenzidine in $20 \mathrm{ml}$ Tris buffer), which was prepared on the day of procedure. $\mathrm{H}_{2} \mathrm{O}_{2}(0.010 \mathrm{ml})$ was added to the activating solution immediately before use. All chemicals were from Sigma. The cells were washed in PBSTween $\left(0.3 \mathrm{ml} \cdot \mathrm{chamber}^{-1}\right)$ for $10 \mathrm{~min}$, and then a quenching step $\left(0.3 \mathrm{ml} \cdot\right.$ chamber $^{-1}$ of $0.3 \% \mathrm{H}_{2} \mathrm{O}_{2}$ in methanol for 30 $\mathrm{min}$ ) was performed to reduce background staining. The slides were then washed in PBS-Tween for $20 \mathrm{~min}$ and incubated with PBS and 10\% FBS for $20 \mathrm{~min}$. After blotting the slides, they were incubated for $30 \mathrm{~min}$ with $0.2 \mathrm{ml}$ of either anti-type I collagen, anti-type III collagen (both Southern Biotech, Birmingham, AL, USA) 
( $n=10$ for each subpopulation), or anti-Kappa chain (control antibody, gift from J. Eldridge) $(n=4)$. All antibodies were biotin-tagged and were diluted 1:1000 in PBS. The cells were washed, incubated for $15 \mathrm{~min}(3$ drops chamber-1 of Vectastain Elite ABC; Vector Lab., Burlingame, CA, USA), and washed again. Then $0.2 \mathrm{ml}$ of the activating solution was added to each chamber and incubated for $5 \mathrm{~min}$. The slides were blinded and analysed. Five areas of each section were evaluated and the sections scored as either positive or negative, avoiding areas where injury to the monolayer or heaping of the cells was noted.

\section{Electron microscopy (EM)}

Sorted fibroblasts (both Thy1 (+) and (-)) were seeded densely $\left(3 \times 10^{5}\right.$ cells on $0.4 \mu \mathrm{m}^{2}$ surface area $)$ on transwell inserts (type HF, Millicell-HA, Millipore; Bedford, MA, USA) and cultured for 3 days. The cells were then processed for EM as follows: samples were fixed in $2.5 \%$ glutaraldehyde in $0.1 \mathrm{M} \mathrm{CaCO}(10 \mathrm{~min} \times 3)$, postfixed with $1 \%$ osmium tetroxide in $0.1 \mathrm{M} \mathrm{CaCO}(10 \mathrm{~min} \times$ 4), and then stained with $0.25 \%$ tannic acid in $0.05 \mathrm{M}$ $\mathrm{CaCO}$ for $60 \mathrm{~min}$. The cells were rinsed in $1 \% \mathrm{Na}_{2} \mathrm{SO}_{4}$ in $0.1 \mathrm{M} \mathrm{CaCO}(10 \mathrm{~min} \times 2)$, then veronal acetate buffer $(10 \mathrm{~min} \times 3)$, and the bloc stained with $2 \%$ uranyl acetate in $30 \% \mathrm{EtOH}$ overnight at $4^{\circ} \mathrm{C}$. The blocs were then serially dehydrated in $\mathrm{EtOH}(50,70,90,95,100 \%)$ and infiltrated with Spurr's resin (1:1 resin:EtOH mixture for $1 \mathrm{~h}$, then $100 \%$ resin for $2 \mathrm{~h}$ at room temperature). Selected regions of the filter were dissected out and embedded in coffin moulds with $100 \%$ resin for $8-20 \mathrm{~h}$ at $70^{\circ} \mathrm{C}$. Blocs were sectioned using a Reichert ultracut E microtome. Grids were stained with uranyl acetate and Reynolds lead citrate. Images were obtained on a JEOL 100CX electron microscope. Greater than 30 cell-to-cell and cell-matrix interactions were evaluated by transmission EM.

\section{Lipid staining}

Sorted cells were seeded onto sterile glass slides and incubated in standard growth medium with $0.05 \%$ ascorbic acid and $0.05 \% \beta$-aminopropionitrile for $96 \mathrm{~h}$. The slides were then air-dried, fixed in propylene glycol (100\%) for $5 \mathrm{~min}$, and then immersed in Oil Red O solution for $7 \mathrm{~min}$ at $56^{\circ} \mathrm{C}$. The slides were then immersed in propylene glycol for an additional $3 \mathrm{~min}$, briefly washed in distilled water, counterstained with haematoxylin, washed in ammonia water and mounted with Aquamount. Mayonnaise was used as a control.

Six slides.group ${ }^{-1}$ were stained and analysed. The slides were blinded, 100 cells $\cdot$ slide $^{-1}$ were counted at $10 \times$ magnification and subjectively scored $0-4$, using the following scale: $0=$ no lipid droplets (red staining); 1=occasional lipid droplets, occupying less than $25 \%$ of the cytoplasm; $2=$ prominent lipid droplets in less than half of the cytoplasm; $3=$ =prominent lipid droplets occupying over half the cytoplasm; 4=large lipid droplets occupying the majo- rity of the cytoplasm. Only cells at the perimeter of the monolayer were evaluated. The total scores (highest possible 400) were averaged and compared between groups.

\section{Statistical analysis}

The data were analysed using the Mann-Whitney Utest (subjective scoring) and Student's t-test, utilizing NCSS (J. Hintze, Kaysville, UT, USA). A probability (p) of less than 0.05 was accepted as significant.

\section{Results}

Rat lung fibroblasts contain two stable subpopulations separable by Thyl expression

We demonstrated that Thy1 (+) and (-) subpopulations of lung fibroblasts exist in rats. Our FACS data show a bimodal distribution of Thy1 expression, suggesting that these are two distinct populations (fig. 1) as has been found in mice. These subpopulations were present in both LEW and F344 rats. Once the cells had been sorted twice, the populations of cells remained stable ( $>90 \%$ purity) over multiple passages ( $>30$ ) and through storage in liquid nitrogen.

LEW and F344 rats differ in their percentage of Thyl (+) and (-) fibroblasts

The percentage of Thy1 (+) lung fibroblasts in six primary cultures from the F344 rats was 27.9 ( \pm 5.4 SD) $\%$; and from the LEW rats was $15.3( \pm 1.97) \%(\mathrm{p}=0.007)$

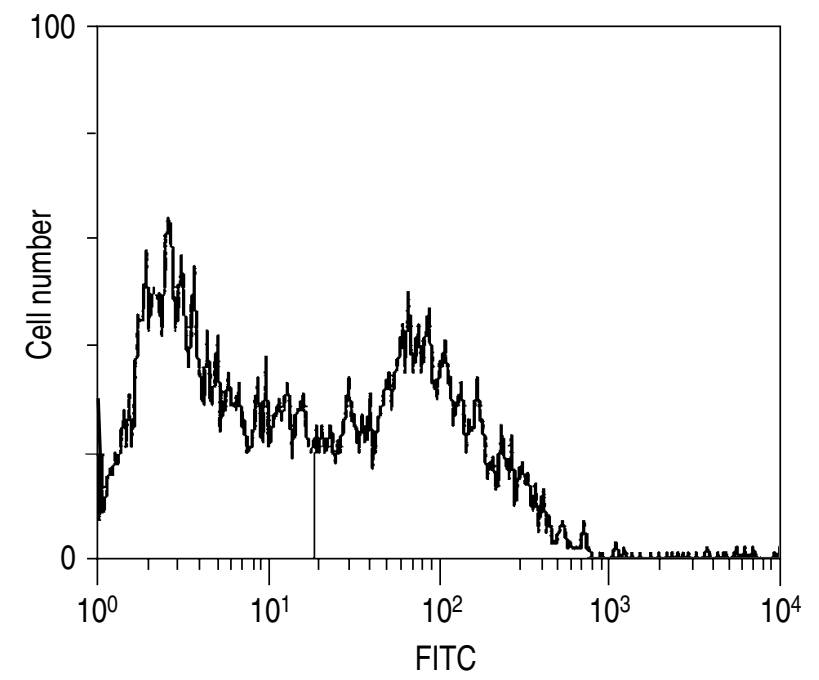

Fig. 1. - A reproduction of a representative sample of a fluorescenceactivated cell sorter (FACS) analysis showing the bimodal distribution of Thy1 expression in primary cultures of these rat lung fibroblasts. The units of the graph are relative, with the y-axis reflecting cell number and the $\mathrm{x}$-axis reflecting relative intensity of fluorescence. FITC: fluorescein isothiocyanate. 


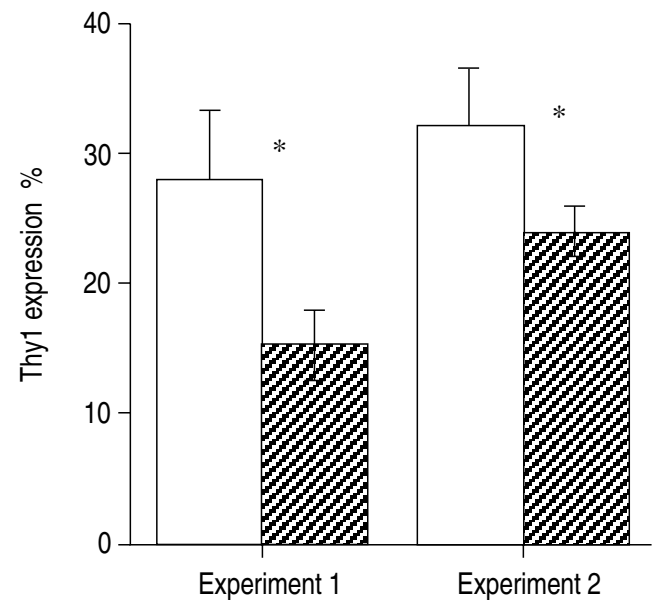

Fig. 2. - The percentage Thy1 (+) fibroblasts found in early passage of two different sets of primary fibroblast cultures ( $\mathrm{n}=6$ from each strain for each experiment) from F344 ( $\square$ ) and LEW ( $\square$ ) rats. Lung fibroblasts from F344 rats had a significantly greater percentage of Thy 1 (+) fibroblasts than those from LEW rats in both experiments. $*: \mathrm{p}<0.01$. Compared to LEW rats

(FACS analysis performed on fibroblasts in their fourth passage). A second experiment with six new primary cultures from LEW and F344 rats was performed (FACS analysis performed on the third passage) and showed similar results (F344 $32.3( \pm 4.42) \%$ vs LEW 24.0
$( \pm 1.96) \%(\mathrm{p}=0.004))$ (fig. 2$)$. Thus, in the naive state, these two rat strains appear to differ in their lung stromal composition, with LEW rats containing a higher percentage of Thy1 (-) fibroblasts than do F344 rats.

\section{Characterization of Thyl (+) and (-) fibroblasts from rat lungs}

Morphology. By light microscopy, the fibroblast subpopulations have different morphological features. Thy 1 (+) fibroblasts are long, flat, and spindle-shaped, whereas Thy1 (-) cells are shorter and more rounded.

Using transmission electron microscopy, multiple cells of each subpopulation were visualized, with particular emphasis upon the area of interaction of cell to grid surface and cell-to-cell. No basal laminae were noted. In addition, no tight junctions were found between confluent cells. The cytoplasm of the Thy 1 (+) cells contained abundant lipid bodies, whereas lipid bodies were sparse in the Thy1 (-) cells.

Collagen production. Thy1 (+) and (-) fibroblasts produce both type I and III collagen. All sections from each subpopulation stained with either type I or type III collagen antibody were judged positive. No background
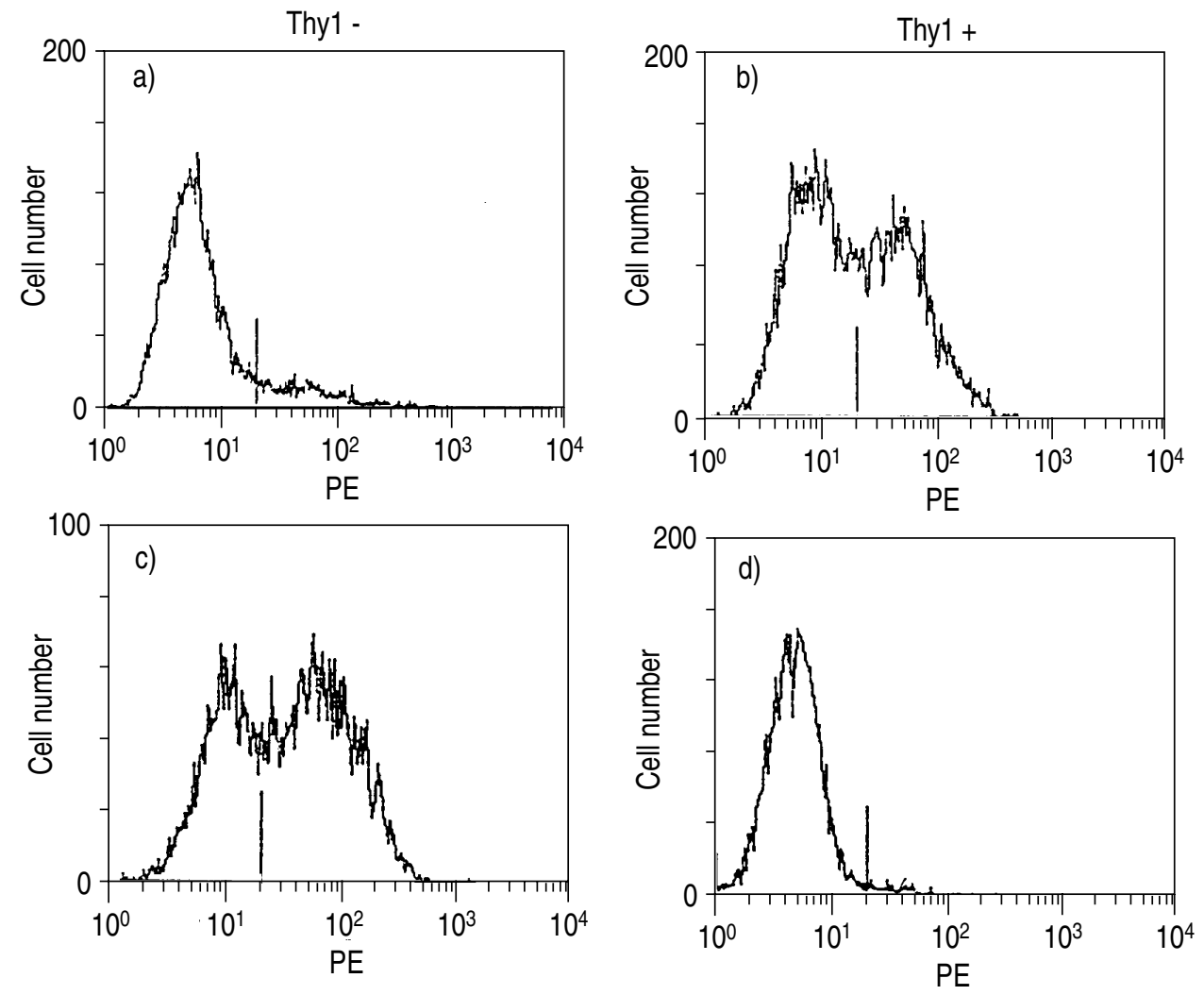

Fig. 3. - A reproduction of representative FACS analyses (one from each group) performed for Ia expression induced by IFN- $\gamma(500$ U.ml-1). There was no constitutive expression noted in any group (i.e in the absence of IFN- $\gamma$ ). a) Ia expression in Thy 1 (-) fibroblasts from naive animals was quite low, but still clearly bimodal. b) A high percentage of Thy1 (+) fibroblasts from naive animals showed Ia expression. c) Thy1 (-) fibroblasts from animals receiving BLM 5 days before sacrifice showed the highest levels of Ia expression. d) Thy1 (+) fibroblasts from BLM treated animals exposed to IFN- $\gamma$ appeared to have a marginally higher percentage of cells expressing Ia than nonexposed, though there is no convincing bimodal expression in these cells. FACS: fluorescence-activated cell sorter; Ia) immune region-associated antigen; IFN- $\gamma$ : interferon$\gamma$; BLM: bleomycin sulphate; PE: phycoerythrin. 
staining was noted using an irrelevant antibody (antiKappa chain) at the same concentration (controls).

Lipid content. Thy1 (+) fibroblasts contain more intracellular lipid than do Thy1 (-) fibroblasts. Using the scoring system enumerated above (possible score 400), Thy1 (+) fibroblasts scored $61.8( \pm 15.6 \mathrm{SEM})$ vs $10.8( \pm 3.4)$ for Thy1 (-) fibroblasts $(\mathrm{p}<0.01)$.

Ia expression in naive and BLM-exposed Thyl (+) and (-) fibroblasts

There was no constitutive Ia expression found in any of the fibroblast subpopulations (i.e none of the fibroblasts groups expressed Ia unless exposed to IFN- $\gamma$ ). After culture in the presence of IFN- $\gamma\left(500 \mathrm{U} \cdot \mathrm{ml}^{-1}\right.$ for $96 \mathrm{~h}$ ), some degree of Ia expression was seen in each group, though the degree of expression in the Thy $1(+)$ from the BLM-treated rats was marginal. In no group did all the cells express Ia (fig. 3).

Six flasks of fibroblasts from each of the groups were exposed to IFN- $\gamma$ and evaluated for Ia expression. Significant differences were noted between the subpopulations. In the fibroblasts from naive animals, the percentage of Ia expression was higher in Thy 1 (+) fibroblasts $(\mathrm{p}<0.05)$, but the results were the opposite when evaluating the percentage Ia expression in the subpopulations from BLM-exposed rats. Thy1 (-) fibroblasts from BLM recipients had significantly higher expression of Ia than did the Thy1 (+) fibroblasts and exceeded that of all other groups $(\mathrm{p}<0.01)$ (fig. 4$)$.

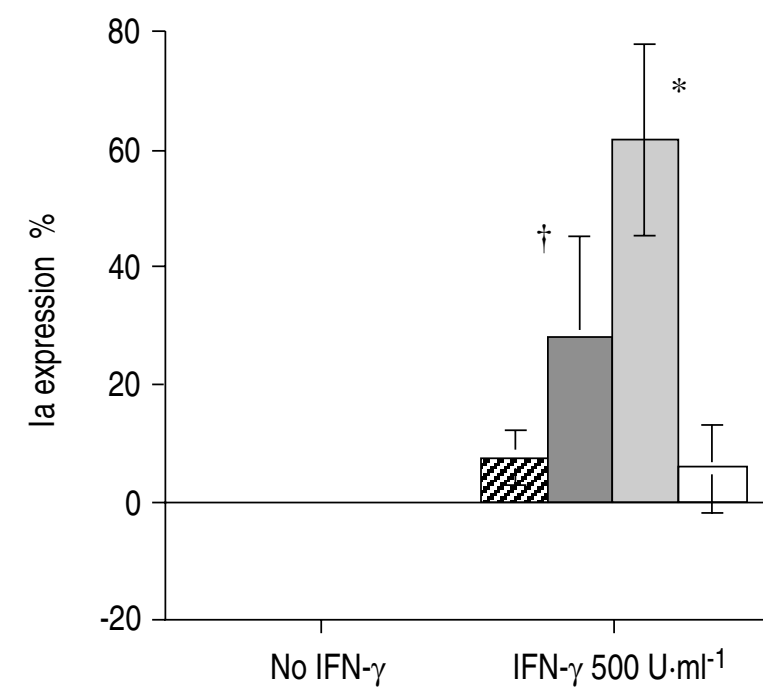

Fig. 4. - Graphic comparison of the mean Ia expression by the fibroblast subpopulations ( $\mathrm{n}=6$ for each group). There was no constitutive expression in any group (i.e. in the absence of IFN- $\gamma$ ). Comparing the IFN- $\gamma$ exposed groups, in fibroblasts from naive rats, Ia expression was significantly higher in Thy1 (+) fibroblasts than in Thy1 (-) fibroblasts. However, in fibroblasts from BLM treated animals, Thy1 (-) fibroblasts showed the highest percentage of Ia expression, and Ia expression in the Thy $1(+)$ was marginal. $\dagger: \mathrm{p}<0.05$ compared to Thy 1 (-) (naive); *: $\mathrm{p}<0.01$ compared to all other groups. $\square$ : Thy $1(-) ; \square$ : Thy $1(+)$; $\square$ : BLM-exposed Thy1 (-); $\square$ : BLM-exposed Thy1 (+). For abbreviations see legend to figure 3 .

\section{Discussion}

These results give additional support to the data presented by PHIPPS et al. [16] in suggesting the importance of Thy $1(+)$ and (-) lung fibroblasts by demonstrating their presence in a second species, by showing that there are differences in the percentage of the fibroblast subpopulations between rat strains with different propensities to fibrosis, and by confirming that the characteristics of the subpopulations are similar between the two species. We demonstrated that in at least two strains of rats, the expression of Thy1 antigen in lung fibroblasts is bimodal, i.e. there are two distinct populations. Moreover, after sorting, those populations remain distinct, with no "reversion to the mean". The distinctive morphology of the two subpopulations is consistent in both species. Thy 1 (+) fibroblasts are long and spindle-shaped; Thy1 (-) fibroblasts are shorter and more rounded. The cytoplasmic lipid content is higher in Thy1 (+) fibroblasts both in rats and mice [18], again demonstrating similarity between the species and raising the question of the relationship between Thy1 subpopulations and foetal fibroblast subpopulations separated by lipid content [22]. These data are very similar to the results from PHIPPS et al. [16] in mice.

We submit that these cells are fibroblasts on the basis of three observations. Firstly, no selective steps were used on the cultured lung tissue. As fibroblasts are abundant and are the hardiest (in vitro) cells in the lungs, lung cell cultures will usually overgrow with fibroblasts unless selective measures are used. Based on culture techniques alone, our cells are probably fibroblasts. Secondly, our cells had the typical morphology of fibroblasts both by light and electron microscopy. Specifically, we demonstrated a lack of tight junctions and basal laminae in cell-cell or cell-matrix interactions, as would be found between cultured endothelial or epithelial cells. Thirdly, these cells produced both type I and type III collagen. Thus, these cells have both positive and negative characteristics suggesting that they are fibroblasts. We cannot clearly differentiate these cells from other cells of mesenchymal origin, such as smooth muscle or myofibroblasts, which are much less abundant, but possess many of the same properties as fibroblasts. We maintain, however, that definitive in vitro differentiation between these various types of mesenchymal cells is not possible at this time [23].

We also showed a significant difference in the percentage of Thy 1 (+) and (-) fibroblast subpopulations in primary cultures from LEW and F344 rats. LEW rats had a higher percentage of Thy1 (-) fibroblasts than did Thy1 (+) fibroblasts in primary cultures from naive animals. There are, admittedly, some problems with these data. There could be differences in the seeding efficiency of the fibroblasts subpopulations between the two strains, though the lungs were handled in an identical manner. There could also be selective growth of one or the other subpopulation after seeding. Our observations so far suggest that the percentage Thy $1(+)$ cells is quite stable over subsequent passages when cells are maintained in log phase growth, as these were. We also acknowledge 
that the differences, though statistically significant, are not enormous. Despite such caveats, these data are important. LEW rats are more susceptible to lung fibrosis than F344 rats following two very different insults. LEW rats develop airway thickness and fibrosis earlier and to a greater extent than do F344 rats after infection with $M$. pulmonis, even though persistent infection after intranasal inoculation is seen in both strains [19]. LEW rats also develop more severe interstitial fibrosis after intratracheal instillation of BLM [20]. These insults differ in the type of injury (infectious versus toxic) and location of injury (airway versus parenchyma), suggesting a difference between the strains in their tendency towards fibrosis. Although, certainly, there is no in vivo proof that Thy1 (+) and (-) fibroblasts differ in their fibrosis promoting potential, the finding that these two strains with differing propensity to fibrosis have different percentages of Thy $1(+)$ and (-) fibroblasts in the naive state suggests an intriguing correlation.

In considering the fibrosis promoting potential of the fibroblast subpopulations, the data concerning Ia expression in rats and mice is important. Ia is the rodent MHC type II antigen. Expression of this antigen by cells not usually involved in antigen presentation, such as fibroblasts, has been suggested as a means by which those cells may participate in regulation of the immune response [24]. The data concerning Ia expression from rats differed from that from mice in naive fibroblasts. PHIPPS et al. [16] showed inducible Ia expression on Thy1 (-) but not Thy $1(+)$ fibroblasts. We showed that in the naive state, both subpopulations could be induced to express Ia, and that a higher percentage of Thy1 (+) than Thy1 (-) fibroblasts expressed Ia. However, following injury with BLM, the pattern was reversed and closely resembled the mice data, with the highest percentage Ia expression of all groups in the BLM-exposed Thy1 (-) fibroblasts, and almost no expression in the BLM-exposed Thy1 (+). This change in Ia expression by Thy1 (-) fibroblasts following a fibrosing insult is in line with evidence given for other cells, such as alveolar macrophages, to support their role in models of inflammatory lung disease [25]. These data add to the emerging picture that fibroblasts are not just responder cells, producing matrix at the beckoning of an inflammatory cell, but are themselves effector cells. Activated lung fibroblasts represent a potential reservoir for upregulating and perpetuating pulmonary inflammation [4].

Fibroblasts are stromal cells, and differences between stromal cell populations have been shown to correlate with differences in experimental disease susceptibility in at least two instances. LEW rats develop more severe experimental allergic encephalomyelitis than do Brown Norway (BN) rats. The stromal cells of the central nervous system, astrocytes, differ in their function between the two rat strains. Stimulated astrocytes from LEW rats produce more tumour necrosis factor (TNF)$\alpha$ than those from $\mathrm{BN}$ rats [26]. Similarly, Wistar rats develop more severe thyroiditis than do F344 rats. Thyroid stromal cells and lung fibroblasts from Wistar rats can be induced to express more Ia than can those from F344 rats [27]. Thus, there is a precedent for the difference in stromal cell populations between rat strains to correlate with differences in disease susceptibility.

In conclusion, Thy1 (+) and (-) fibroblast subpopulations with similar characteristics have now been found in two different species, rats and mice. In both species, primary cultures of lung fibroblasts have a bimodal distribution of Thy 1 antigen expression, which, after sorting, remains stable over multiple passages. Many of the characteristics of the subpopulations are preserved between mice and rats. The major difference between the species is in Ia expression, as discussed above. The presence of Thy1 (+) and (-) fibroblast subpopulations in two species with predominantly preserved characteristics, many of which suggest differences in the propensity of the fibroblasts to interact with the inflammatory cascade, reinforces the potential importance of the subpopulations. In two rat strains (LEW and F344) that differ in susceptibility to lung fibrosis from two different insults, there are significant differences in the percentage of Thy1 (+) and (-) fibroblasts in primary culture from naive animals. We would suggest several areas of study that might prove fruitful in further evaluating the significance of lung fibroblast subpopulations. One area is evaluation of changes in the relative abundance and function of fibroblast subpopulations in disease, such as BLM-induced fibrosis. The difference we reported here in Ia expression between the subpopulations from naive animals and from rats exposed to BLM is a start in these types of studies. Another area would be studies of in vitro response to growth modulators. Differences found here would further suggest the possibility that the subpopulations do not act in the same way in fibrotic lung diseases, and that these differences could be exploited in vivo to try to selectively alter fibroblast proliferation. Finally, studies of the bidirectional interactions of fibroblast subpopulations with various relevant cells (alveolar macrophages, lymphocytes, type II epithelial cells) will be needed to determine the different roles that fibroblast subpopulations play in development and disease.

Acknowledgements: The authors wish to thank E. Philips and D. Vintor for their assistance with the electron microscopy, and J.R. Wright for her criticisms of the manuscript.

\section{References}

1. Crystal RG, Bitterman PB, Rennard SI, Hance AJ, Keogh BA. Interstitial lung diseases of unknown cause: disorders characterized by chronic inflammation of the lower respiratory tract (2 parts). N Engl J Med 1994; 310: 154-166 and 235-244.

2. Polunovsky VA, Chen B, Henke C, et al. Role of mesenchymal cell death in lung remodeling after injury. J Clin Invest 1993; 92: 388-397.

3. Elias JA, Trinchieri G, Beck JM, et al. A synergistic interaction of IL- 6 and IL- 1 mediates the thymocytestimulating activity produced by recombinant IL-1 stimulated fibroblasts. J Immunol 1989; 142: 509-514.

4. Streiter RM, Phan SH, Remick DG, et al. Monokineinduced neutrophil chemotactic factor gene expression in human fibroblasts. J Biol Chem 1989; 264: 1062110626. 
5. Adamson IYR, Hedgecock C, Bowden DH. Epithelial cell-fibroblast interactions in lung injury and repair. $\mathrm{Am}$ J Pathol 1990; 137: 385-392.

6. Smith DT, Post M. Fibroblast-pneumocyte factor. Am J Physiol (Lung Cell Mol Physiol) 1989; 257: L174L178.

7. Scott S, Pandolfi F, Kurnick JT. Fibroblasts mediate Tcell survival: a proposed mechanism for retention of primed T-cells. J Exp Med 1990; 172: 1873-1876.

8. Vancheri C, Gauldie J, Bienenstock J, et al. Human lung fibroblast-derived granulocyte-macrophage colony stimulating factor (GM-CSF) mediates eosinophil survival in vitro. Am J Respir Cell Mol Biol 1989; 1: 289295.

9. Korn JH, Torres D, Downie E. Clonal heterogeneity in the fibroblast response to mononuclear cell derived mediators. Arthritis Rheum 1984; 27: 174-179.

10. Botstein GR, Sherer GK, LeRoy EC. Fibroblast selection in scleroderma: an alternative model of fibrosis. Arthritis Rheum 1982; 25: 189-195.

11. Raghu G, Chen Y, Rusch V, Rabinovitch PS. Differential proliferation of fibroblasts cultured from normal and fibrotic human lungs. Am Rev Respir Dis 1988; 136: 703-708.

12. Breen E, Falco VM, Absher M, Cutroneo KR. Subpopulations of rat lung fibroblasts with different amounts of type I and type II collagen mRNAs. J Biol Chem 1990; 265: 6286-6290.

13. Caniggia I, Tanswell K, Post M. Temporal and spatial differences in glycosaminoglycan synthesis by fetal lung fibroblasts. Exp Lung Res 1992; 202: 252-258.

14. Brody JS, Kaplan NB. Proliferation of alveolar interstitial cells during postnatal lung growth. Am Rev Respir Dis 1983; 127: 763-770.

15. Akamine A, Raghu G, Narayanan AS. Human lung fibroblast subpopulations with different C1q binding and functional properties. Am J Respir Cell Mol Biol 1992; 6: 382-389.

16. Phipps RP, Penney DP, Keng P, et al. Characterization of two major populations of lung fibroblasts: distinguishing morphology and discordant display of Thy 1 and class II MHC. Am J Respir Cell Mol Biol 1989; 1: 65-74.

17. Williams AF, Barclay AN. The immunoglobulin superfamily-domains for cell surface recognition. Ann Rev Immunol 1988; 6: 381-405.

18. Penney DP, Keng PC, Derdak S, Phipps RP. Morphologic and functional characteristics of subpopulations of murine lung fibroblasts grown in vitro. Anat Rec 1992; 232: 432-443.

19. Phipps RP, Baecher C, Frelinger JG, Penny DP, Keng $\mathrm{P}$, Brown D. Differential expression of interleukin-1 $\alpha$ by Thy $1(+)$ and (-) lung fibroblast subpopulations: enhancement of interleukin- $1 \alpha$ production by tumor necrosis factor- $\alpha$. Eur J Immunol 1990; 20: 1723-1727.

20. McIntosh JC, Simecka JW, Ross SE, Davis JK, Miller EJ, Cassell GH. Infection-induced airway fibrosis in two rat strains with differential susceptibility. Infect Immun 1992; 60: 2936-2942.

21. McIntosh JC, Liang SC, Simecka JW, et al. Differential fibrosis in two rat models. Chest 1989; 96: 172S.

22. Maksvytis HJ, Niles RM, Simanovsky L, et al. In vitro characteristics of the lipid filled interstitial cell associated with postnatal lung growth: evidence for fibroblast heterogeneity. J Cell Physiol 1984; 118: 113-123.

23. Mitchell JJ, Woodcock-Mitchell JL, Perry L, et al. In vitro expression of the $\alpha$-smooth muscle actin isoform by rat lung mesenchymal cells: regulation by culture condition and transforming growth factor- $\beta$. Am J Respir Cell Mol Biol 1993; 9: 10-18.

24. Holt PG. Regulation of antigen-presenting cell function(s) in the lung and airway tissue. Eur Respir J 1993; 6: $120-129$.

25. Kaltreider HB, Byrd PK, Curtis JL. Expression of Ia by murine alveolar macrophages is upregulated during the evolution of a specific immune response in pulmonary parenchyma. Am Rev Respir Dis 1988; 137: 14111416.

26. Chung IY, Norris JG, Benveniste EN. Differential tumor necrosis factor- $\alpha$ expression by astrocytes from experimental allergic encephalomyelitis-susceptible and resistant rat strains. J Exp Med 1991; 173: 801-811.

27. Neufeld DS, Davies TF. Strain-specific determination of the degree of thyroid cell MHC class II antigen expression: evaluation of established Wistar and Fisher rat thyroid cell lines. Endocrinol 1990; 127: 12541259. 